\title{
Prediction of long-term settlement and accurate analysis of horizontal displacement based on model test results
}

\author{
Toshifumi Shibata ${ }^{\text {i) }}$, Shin-ichi Nishimura ${ }^{\text {ii) }}$ and Takayuki Shuku ${ }^{\text {iii) }}$ \\ i) Associate professor, Graduate School of Environmental and Life Science, Okayama University, 3-1-1 Tsushima-Naka, Kita-ku, \\ Okayama 700-8530, Japan. \\ ii) Professor, Graduate School of Environmental and Life Science, Okayama University, 3-1-1 Tsushima-Naka, Kita-ku, \\ Okayama 700-8530, Japan. \\ iii) Research associate, Graduate School of Environmental and Life Science, Okayama University, 3-1-1 Tsushima-Naka, Kita-ku,
} Okayama 700-8530, Japan.

\begin{abstract}
This paper presents long-term predictions of the ground behavior, including secondary consolidation via the particle filter, using model test results. There have been some difficulties in evaluating lateral displacements, because an unsuitable value for Poisson's ratio and the constitutive model are generally employed for consolidation simulations. In this paper, the Poisson's ratio is identified in addition to the compression index, the permeability, the initial volumetric strain, and the secondary compression index in the modified Cam-clay model considering anisotropy, to achieve more accurate predictions of lateral displacements. The prediction is performed using the identified parameters, and numerical examples of accurate predictions of the ground behavior are shown.
\end{abstract}

Keywords: particle filter, consolidation, prediction, horizontal displacement

\section{INTRODUCTION}

The numerical prediction of long-term deformation behavior of the ground under earth structures is of the utmost importance due to maintenance concerns. Normally, the parameters in the simulation are evaluated by means of laboratory and in situ tests. However, due to various uncertainties, it is not easy to obtain appropriate soil parameters based purely on such tests. An inverse analysis is a powerful tool for reevaluating the parameters based on measurements to overcome the many sources of uncertainty. The particle filter (henceforth, PF) (Gordon, et al., 1993), one of the methods of inverse analysis, is advantageous for nonlinear problem such as the simulation of geotechnical problems (Shuku et al., 2012; Murakami et al., 2013; Shibata et al., 2014). We have reported the identification of elasto-plastic parameters in terms of secondary consolidation, based on the results of model tests by means of the PF, and the prediction of the long-term behavior by using the identified parameters (Nishimura et al., 2013). An accurate prediction of the long-term settlement was presented; however, a less accurate computation of the lateral deformation was shown. This is because the constitutive model tends to overestimate the horizontal displacement and this leads to the adoption of an unsuitable value for Poisson's ratio. This paper

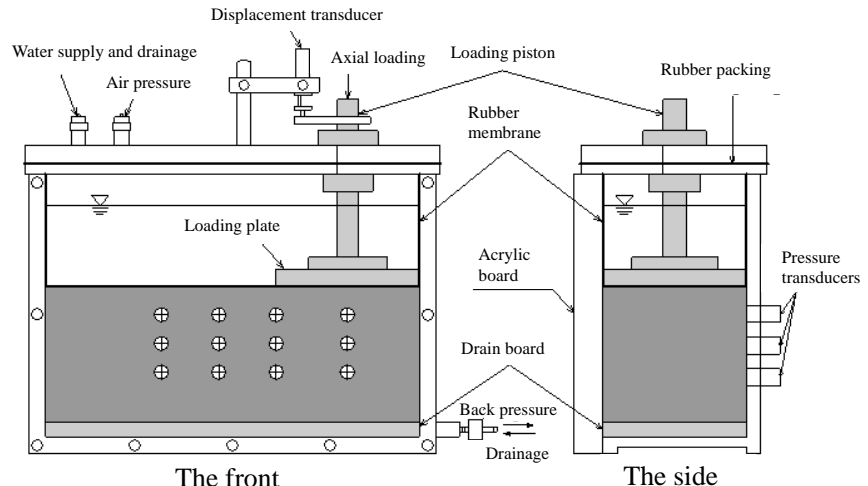

Fig. 1. Model test apparatus (Shuku et al., 2012).

presents the long-term prediction of the ground behavior including secondary consolidation by an elasto-plastic FEM via the PF using the model test results. The Poisson's ratio, along with the compression index, the permeability, the initial volumetric strain, and the secondary compression index, are identified by the PF. The modified Cam-clay model, considering anisotropy, is employed to improve the prediction accuracy of horizontal displacement. Predictions using the identified parameters are 
Table 1. Physical properties (Nishimura et al., 2013).

\begin{tabular}{c|c|c|c|c|c}
\hline$\rho\left(\mathrm{g} / \mathrm{cm}^{3}\right)$ & $W_{L}(\%)$ & $W_{P}(\%)$ & $I_{P}$ & $\begin{array}{c}\text { Clay } \\
\text { fraction } \\
(\%)\end{array}$ & $\begin{array}{c}\text { Silt } \\
\text { fraction } \\
(\%)\end{array}$ \\
\hline 2.694 & 102.0 & 30.6 & 71.4 & 55.0 & 45.0 \\
\hline
\end{tabular}

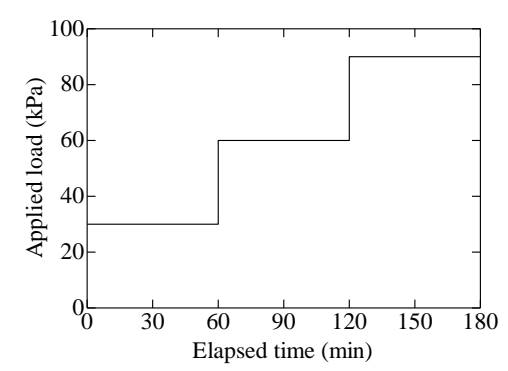

Fig.2. Loading process (Nishimura et al., 2013).

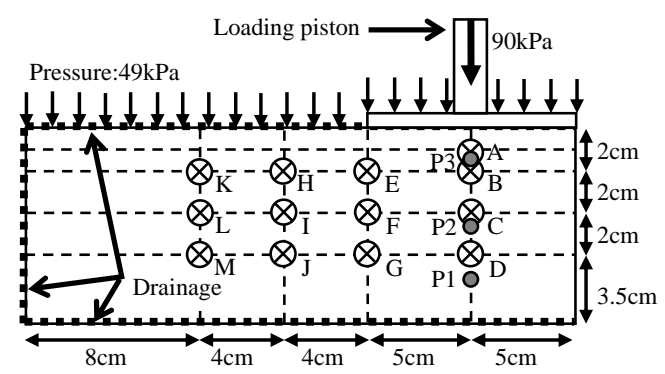

Fig. 3. Placement of measurement instruments (Nishimura et al., 2013).

Table 2. Parameter values.

\begin{tabular}{c|c}
\hline$M$ & 0.941 \\
$\sigma_{v}^{\prime}\left(\mathrm{kN} / \mathrm{m}^{2}\right)$ & 49.0 \\
$K_{0}$ & 0.740 \\
$\sigma_{v i}^{\prime}\left(\mathrm{kN} / \mathrm{m}^{2}\right)$ & 49.0 \\
$K_{i}$ & 0.740 \\
$e_{0}$ & 2.049 \\
\hline
\end{tabular}

performed, and some examples of accurate predictions of the ground behavior are shown.

\section{MODEL TEST APPARATUS}

Fig. 1 shows the model test apparatus which consists of a container box, a rigid footing, a loading piston, and several controllers. The front wall of the container box was made of a transparent acrylic plate to monitor the deformation of the soil block sample. The sample, whose physical properties are listed in Table 1, was loaded partially by the piston while constant confining pressure was applied to the surface through a rubber membrane. In Table $1, \rho$ is the density of the soil, WL is the liquid limit, $W_{P}$ is the plastic limit, and $I_{P}$ is the plasticity index. The soil block sample was created at a preliminary consolidation pressure of $49 \mathrm{kPa}$, and the process of the partial load is shown in Fig. 2. To observe the deformation and the pore water

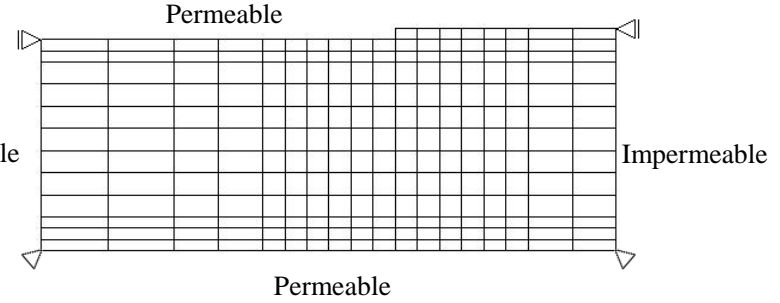

Fig. 4. Finite element mesh and boundary conditions.

Table 3. Feasible space for parameters.

\begin{tabular}{c|c|c}
\hline & Case 1 & Case 2 \\
\hline$\lambda$ & $0.150 \sim 0.450$ & $0.200 \sim 0.500$ \\
$\log k$ & $-8.28 \sim-7.94$ & $-8.18 \sim-7.84$ \\
$\alpha$ & $2.50 \times 10^{-3} \sim 3.90 \times 10^{-3}$ & $1.20 \times 10^{-3} \sim 2.80 \times 10^{-3}$ \\
$\dot{v}_{0}(1 / \mathrm{min})$ & $0 \sim 1.18 \times 10^{-7}$ & $0 \sim 1.18 \times 10^{-7}$ \\
$v$ & $2.00 \times 10^{-2} \sim 2.80 \times 10^{-1}$ & $2.00 \times 10^{-2} \sim 1.80 \times 10^{-1}$ \\
\hline
\end{tabular}

Table 4. Parameter values finally identified.

\begin{tabular}{c|c|c}
\hline & Case-1 & Case- 2 \\
\hline$\lambda$ & 0.245 & 0.327 \\
$k \quad(\mathrm{~m} / \mathrm{min})$ & $6.67 \times 10^{-9}$ & $1.08 \times 10^{-8}$ \\
$\dot{v}_{0}(1 / \mathrm{min})$ & $4.08 \times 10^{-8}$ & $5.08 \times 10^{-8}$ \\
$\alpha$ & $3.41 \times 10^{-3}$ & $2.22 \times 10^{-3}$ \\
$\nu$ & 0.0971 & 0.0941 \\
\hline
\end{tabular}

pressure of the soil block sample, displacement- tracing pointers (A-M) and pressure transducers (P1-P3) were installed, as presented in Fig. 3, and the movements of the pointers were recorded with a digital video camera. Ten displacement devices among the thirteen and two pressure transducers among the three were used to identify the soil parameters.

\section{SIMULATION}

The geotechnical parameters listed in Table 3 are identified by the PF with Sequential Importance Sampling (SIS) (Doucet et al., 2000) based on the observation data such as the settlement, the lateral displacement, and the pore water pressure. The soil-water coupled FEM with an elasto-plastic constitutive model, DACSAR (Iizuka and Ohta, 1987), was used as the numerical simiulation method. The identified parameters were used as the input parameters for the FEM, and the prediction results are compared to the corresponding observation data to check the simulation accuracy.

This paper presents the following three cases of identification to examine the accuracy of the numerical results. 

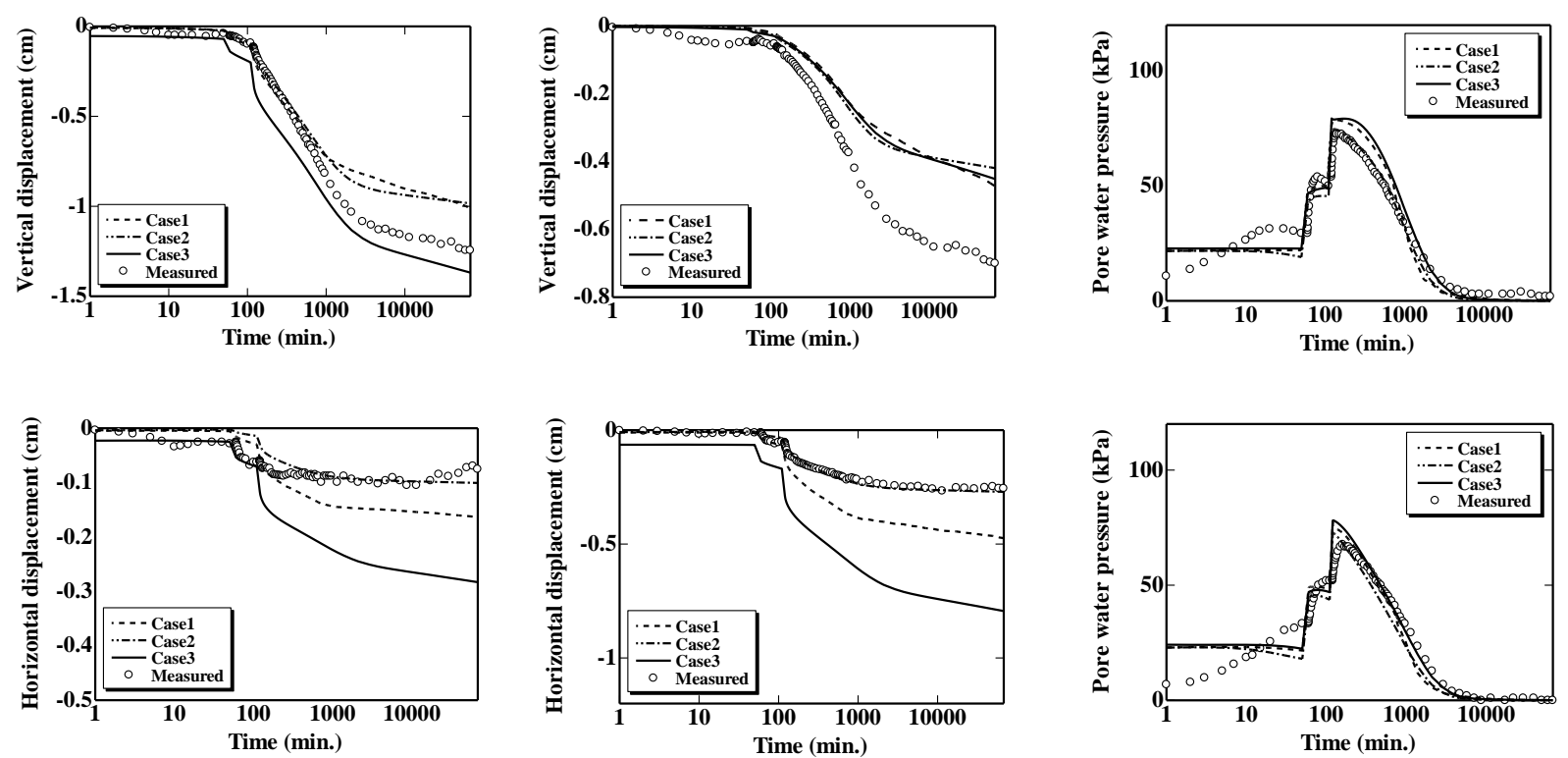

(a) Vertical and horizontal displacements at Points A and E.

(b) Pore water pressure at Points P2 and P3. Fig. 5. Comparison between direct analysis using identified parameters and corresponding measures.
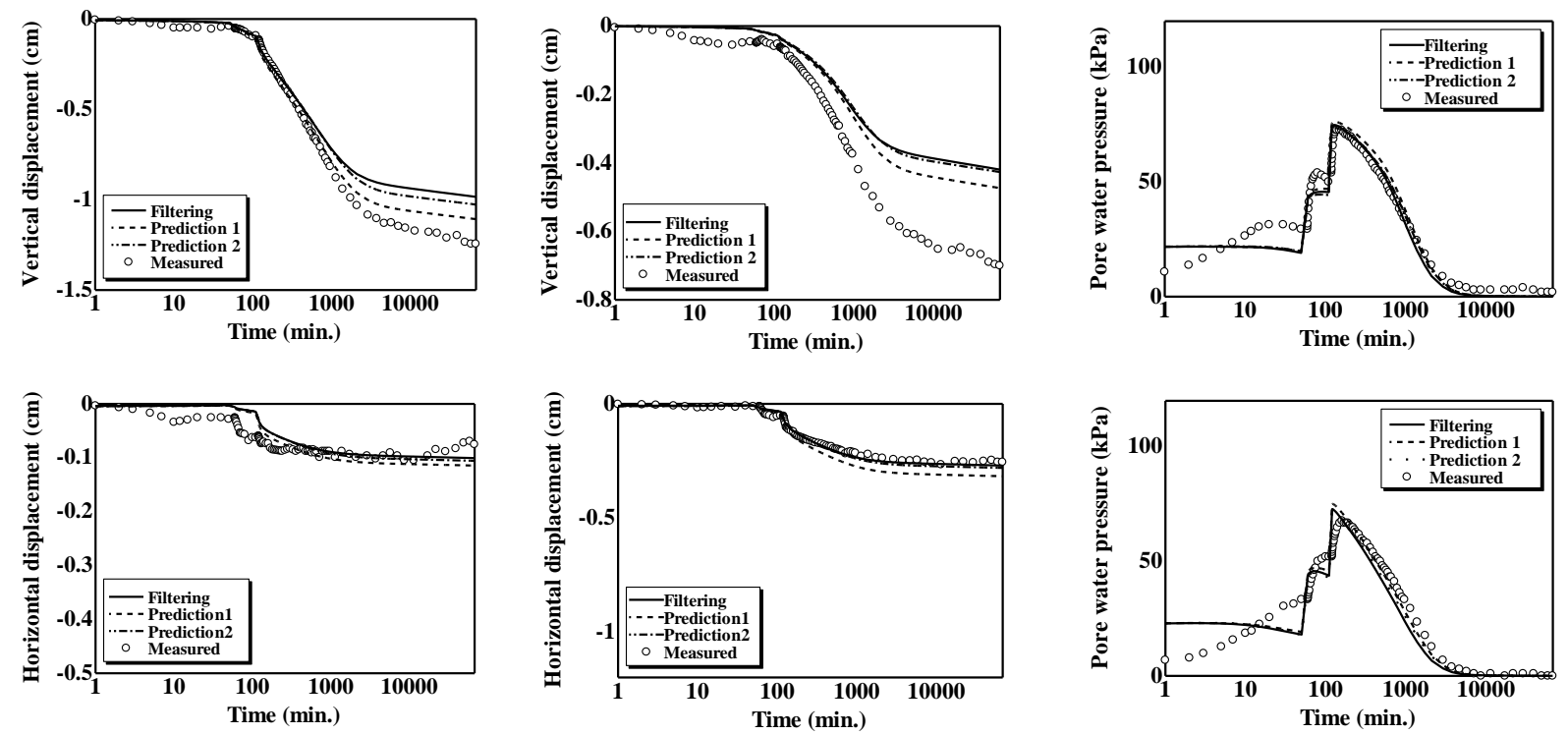

(a) Vertical and horizontal displacements at Points A and E.

(b) Pore water pressure at Points P2 and P3.

Fig. 6. Comparison between predicted results using identified parameters and corresponding measures.

a) Case-1: The Sekiguchi-Ohta model (viscid version) (Seckiguchi and Ohta, 1977) is employed as the constitutive model and five soil parameters are identified, i.e., Poisson's ratio, the compression index, the permeability, the initial volumetric strain, and the secondary compression index.

b) Case-2: The modified Cam-clay model, considering anisotropy, is adopted. The soil parameters to be identified are the same as in Case-1.

c) Case-3: The constitutive model is the same as in Case-1, and all the soil parameters are identified, except Poisson's ratio. A Poisson's ratio of 0.425 is used.

Fig. 4 depicts the adopted finite element model consisting of eight-node isoparametric quadrilateral elements with full integration and boundary conditions. Table 2 lists the soil parameters in which $M$ is the critical state parameter, $\sigma_{v}$ is the preconsolidation pressure, $K_{0}$ is the coefficient of in situ earth pressure, $\sigma_{\text {vi }}^{\prime}$ is the effective overburden pressure in situ, $K_{\mathrm{i}}$ is the coefficient of in situ earth pressure at rest, and $e_{0}$ is the void ratio corresponding to the preconsolidation 
pressure at the reference state. The settlement, the horizontal displacement, and the pore water pressure are used as the observation data for the PF from 160 minutes. 1000 sets of particles are generated as uniform random numbers within the range tabulated in Table 3. The diagonal term for the observation error covariance matrix, $\sigma^{2}$, is assumed to be 1.0 in terms of vertical and horizontal displacements, and $\sigma^{2}$ of 1000 and 500 are adopted with respect to the pore water pressure at $\mathrm{P} 2$ and $\mathrm{P} 3$, respectively.

\section{NUMERICAL RESULTS}

Table 4 lists the identified parameters using the PF, and Fig. 5 compares the simulated results using the identified parameters with the measurements. In Case-1, the measurements for the horizontal displacements at Points $\mathrm{A}$ and $\mathrm{E}$ are slightly smaller than the corresponding numerical results, whereas those of the vertical displacements are larger than the results of the analysis, as presented in Fig. 5 (a). In Case-2, the measured horizontal displacements are quite close to the simulation results; however, the measurements in Case-3 deviate somewhat from the calculated results. It is clear, therefore, that both an appropriate Poisson's ratio and the use of the modified Cam-clay model, taking into account the anisotropy, are necessary for accurately evaluating the lateral displacement. In all cases, the measurements of pore water pressure at Points P2 and P3 match the simulation results well, as shown in Fig. 5 (b).

Fig. 6 illustrates the prediction of the displacements at Points $\mathrm{A}$ and $\mathrm{E}$, and the pore water pressure at Points P2 and P3. In Fig. 6, the prediction, using the identified parameters obtained from the measurements from 160 minutes to the end of the experiment, is denoted as "Filtering". The prediction via the measurements from 160 minutes to 7088 minutes is called "Prediction 1", and the prediction using the measurements from 160 minutes to 840 minutes is denoted as "Prediction 2". The measurements of the horizontal displacements and the pore water pressure match the results of the prediction well, although the measured vertical displacements differ slightly from the predicted displacements. From these figures, it can be interrupted that successful predictions of the displacement and the pore water pressure are presented for all cases.

\section{CONCLUSIONS}

This paper has presented a prediction of the vertical and horizontal displacements, and the pore water pressure, in conjunction with a soil-water coupled FEM using the PF through measured data. The PF was employed to identify the soil parameters, and the identified parameters were used for the prediction. Poisson's ratio, along with the compression index, the permeability, the initial volumetric strain, and the secondary compression index, was identified, while the modified Cam-clay model, considering anisotropy, was adopted to improve the accuracy of the numerical results of the horizontal displacements.

The simulation results for the horizontal displacements showed a good agreement with the corresponding measurement data, although there were slight differences between the results of the analysis and the measured settlements and the pore water pressure. To predict the ground behavior, computation via the identified parameters was carried out. The simulation results of the horizontal displacements were found to be close to the actual measurement data. Hence, the simulations of the lateral displacements with the modified Cam-clay model considering anisotropy, using the identified parameters including Poisson's ratio, yielded predictions with high accuracy.

\section{REFERENCES}

1) Doucet, A., Godsill, S. and Andrieu, C. (2000): On sequential Monte Carlo sampling methods for Bayesian filtering, Statistics and Computing, 10, 197-208.

2) Gordon, N. J., Salmond, D. J. and Smith, A. F. M. (1993): Novel approach to nonlinear/non-Gaussian Bayesian state estimation, IEE Proceedings-F, 140(2), 107-113.

3) Iizuka, A. and Ohta, H. (1987): Determination procedure of input parameters in elasto-viscoplastic finite element analysis, Soils and Foundations, 27(3), 71-87.

4) Murakami, A., Shuku, T., Nishimura, S., Fujisawa, K. and Nakamura, K. (2013): Data assimilation using the particle filter for identifying the elasto-plastic material properties of geomaterials, International Journal for Numerical and Analytical Methods in Geomechanics, 37(11), 1642-1669.

5) Nishimura, S., Shuku, T., Yamada, N. and Shibata, T. (2013): Verification of long-term settlement prediction method based on model test results, Journal of JSCE A2, 69(2), I_29-I_38, (in Japanese).

6) Sekiguchi, H. and Ohta, H. (1977): Induced anisotropy and time dependency in clays, Proceedings of Specialty Session 9, 9th International Conference of Soil Mechanics and Foundation Engineering, 229-239.

7) Shibata, T., Murakami, A. and Fujii, M. (2014): Prediction of embankment behavior of regulating reservoir with foundation improved by vacuum consolidation method, Soils and Foundations, 54(5), 938-954.

8) Shuku, T., Murakami, A., Nishimura, S., Fujisawa, K. and Nakamura, K. (2012): Parameter identification for Cam-clay model in partially loading tests using the particle filter, Soils and Foundations, 52(2), 279-298. 